\title{
Nanomechanical strength mechanisms of hierarchical biological materials and tissues
}

\author{
Markus J. Buehler* and Theodor Ackbarow
}

Laboratory for Atomistic and Molecular Mechanics, Department of Civil and Environmental Engineering, Massachusetts Institute of Technology, Cambridge, MA, USA

(Received 22 August 2007; final version received 15 March 2008)

Biological protein materials (BPMs), intriguing hierarchical structures formed by assembly of chemical building blocks, are crucial for critical functions of life. The structural details of BPMs are fascinating: They represent a combination of universally found motifs such as $\alpha$-helices or $\beta$-sheets with highly adapted protein structures such as cytoskeletal networks or spider silk nanocomposites. BPMs combine properties like strength and robustness, self-healing ability, adaptability, changeability, evolvability and others into multi-functional materials at a level unmatched in synthetic materials. The ability to achieve these properties depends critically on the particular traits of these materials, first and foremost their hierarchical architecture and seamless integration of material and structure, from nano to macro. Here, we provide a brief review of this field and outline new research directions, along with a review of recent research results in the development of structureproperty relationships of biological protein materials exemplified in a study of vimentin intermediate filaments.

Keywords: hierarchical; nanomechanics; biological protein materials; fracture; deformation; experiment; simulation; vimentin; cytoskeleton

\section{Introduction}

Proteins are the fundamental building blocks of a vast array of biological materials that are involved in critical functions of life, many of which are based on highly characteristic nanostructured arrangements of protein components that include tropocollagen, $\alpha$-helices or $\beta$-sheets (Lakes 1993; Alberts et al. 2002; Wegst and Ashby 2004; Aizenberg et al. 2005). Bone, providing structure to our body, or spider silk, used for prey procurement, are examples of materials that have incredible elasticity, strength and robustness unmatched by many synthetic materials (Buehler 1961; Hayashi and Lewis 1998; Weiner and Wagner 1998; Arzt et al. 2003; Sarikaya et al. 2003; Buehler 2006a, 2006b; Buehler and Ackbarow 2007).

Biological protein materials (BPMs) are particularly fascinating as they combine properties such as strength and robustness, self-healing ability, adaptability, changeability, evolvability and many other properties into multifunctional materials at a level still unmatched in synthetic materials. It is believed that the ability to achieve these properties depends critically on the particular traits of these materials, first and foremost their hierarchical architecture and the seamless integration of material and structure, from nano to macro.

Here, we review recent advances in utilising largescale atomistic and molecular modeling that elucidate the deformation and fracture mechanisms of BPMs. We illustrate how a combination of predictive atomistic modelling implemented on large scale computing facilities and theoretical analysis using statistical and continuum theories enables us to carry out highly spatially and temporally controlled experiments to probe the behaviour of protein structures at different hierarchical scales, ranging from single proteins, assemblies of proteins to protein networks, at time scales that range from picoseconds, nanoseconds to microseconds. The systematic traversing across multiple scales enables us to elucidate the scale-dependent properties and enables us to develop structure-property relationships. Clearly, this research strategy must be based on a multidisciplinary approach that combines chemistry, biology and materials science.

The particular focus of the present work is on BPMs that play a mechanical role in physiological systems (Engler et al. 2006). This is motivated by the emergence of evidence that mechanical cues are central to many biological processes, as for instance in cell differentiation, mechanosensation/transduction, amyloidosis or cellular viral capsid injection (Alberts et al. 2002; Engler et al. 2006). In some of these processes, mechanical cues directly influence gene regulation that then trigger biochemical signalling cascades that lead to a nonlocal response, controlling the behaviour of larger tissues scales. A particularly important field is thereby the analysis of the nanomechanical properties of participating proteins and protein networks, by describing the processes and behaviours when BPMs are stretched, bent or loaded in compression, and therefore undergo significant changes in shape and molecular structure.

\section{*Corresponding author. Email: mbuehler@mit.edu}


Theories that describe these mechanical processes and properties must explicitly consider the chemical bonding and molecular structure, due to the strong interaction of chemistry and mechanics (Buehler 2007). In addition, biological processes often involve mechanical properties that are controlled by chemical cues (Alberts et al. 2002). This combination is the equivalent of the extension of systems biology towards the ultimate material nano-scale at the boundary of chemistry, biology and materials science (Ackbarow and Buehler). This could help to answer important open questions in the biological sciences, such as the elucidation of the boundaries and characteristics that distinguish a 'material' and 'living organism'.

\subsection{Materials science of biological materials}

Historically, the use of classes of materials has been used classify stages of civilisations, ranging from stone age more than 300,000 years ago, to the bronze age, and possibly the silicon age in the late 20 th and early 21 st century. However, a systematic analysis of materials in the context of linking chemical and physical concepts with engineering applications has not been achieved until quite recently. For instance, 50 years ago, E. Orowan, M. Polanyi and G.I. Taylor discovered dislocations, a concept proposed theoretically in 1905 by V. Volterra. It was discovered that dislocations represent the fundamental mechanism of plastic deformation of metals and other ductile, crystalline materials (Taylor 1934; Hirth and Lothe 1982). Remarkably, it was not until dislocations and other nano- and microscopic mechanisms have been understood theoretically that major breakthroughs have been possible that utilise this knowledge, to enable building airplanes, cars, space shuttles and more recently, nanodevices, through synthesis of ultra-strong and heat resistant materials, for instance (Buehler 2007).

Perhaps, today we stand at a similar cross-road: Biological materials and systems are vital elements of life, and therefore, a rigorous understanding of the matter that makes life 'work' is in reach. This may enable us eventually to integrate concepts from living systems into materials design, seamlessly. Optical, mechanical and electrical properties at ultra-small material scales, their control, synthesis and analysis as well as their theoretical description represent major scientific and engineering challenges and opportunities. However, just like in the case of more conventional materials, these breakthroughs will probably only be accessible provided that the fundamentals are well understood. Characterisation of the materials found in biology within a rigorous materials science approach is aimed towards the elucidation of these fundamental principles of assembly, deformation and fracture of these materials.

Deformation and fracture properties are intimately linked to the atomic microstructure of the material. Whereas crystalline materials show mechanisms such as dislocation nucleation and growth or crack extension, biological materials feature molecular unfolding or intermolecular sliding, with a particular significance of rupture of chemical bonds such as hydrogen bonds (the focus of this article), covalent cross-links or intermolecular entanglement. Much different mechanisms operate at larger length scales, where the interaction with cells, and of cells with one another, different tissue types and the influence of tissue remodelling become more evident. The dominance of specific mechanisms is controlled by geometric parameters as well as the structural arrangement of the protein's elementary building blocks, across many hierarchical scales, from nano to macro (Figure 1).

It is known from other fields in materials science that nano- or microscopic structures control the macroscopic material behaviour: For example, grain size reduction or confinement leads to an increase of the strength of crystalline metals (Nieh and Wadsworth 1991; Yip 1998; Blanckenhagen et al. 2001; Wolf et al. 2003) (Hall-Petch effect) and a reduction of strength at even smaller scales (inverse Hall-Petch effects). Deformation maps have been proposed to characterise material properties for engineering applications (Frost and Ashby 1982). Discovering similar insight into the scaling behaviour, and the understanding of how structural features control the properties of biological structures and materials represents and important frontier of research. A particularly challenging question is the elucidation of the significance and the role of nanostructures for macroscopic properties.

A major trait of biological materials is the occurrence of hierarchies and, at the molecular scale, the abundance of weak interactions such as H-bonds. The presence of hierarchies in biological materials may be vital to take advantage of molecular and sub-molecular features, often characterized by weak interactions, and enhance their properties so that they become more proncounced at larger scales, in order to provide a link between structural organisation and function (Fraser and Bickmore 2007). Utilisation of weak interactions makes it possible to synthesise strong materials at moderate temperatures and thus with limited energy use, albeit at possibly longer time scales (biological systems assemble at time scales from seconds to years). Clearly, this calls for the development of new methods that provide more rapid synthesis of such materials. An important distinction between traditional and biological materials is the geometrical occurrence of defects. While defects are often distributed randomly over the volume in crystalline materials, biological materials consist of an ordered structure that reaches down to the nano-scale. Notably, in many biological materials, defects are appear with atomistic or molecular precision, and may play a major role in the material behaviour observed at larger scales. These features have been observed in bone, nacre, collagenous tissue or cellular protein networks. 


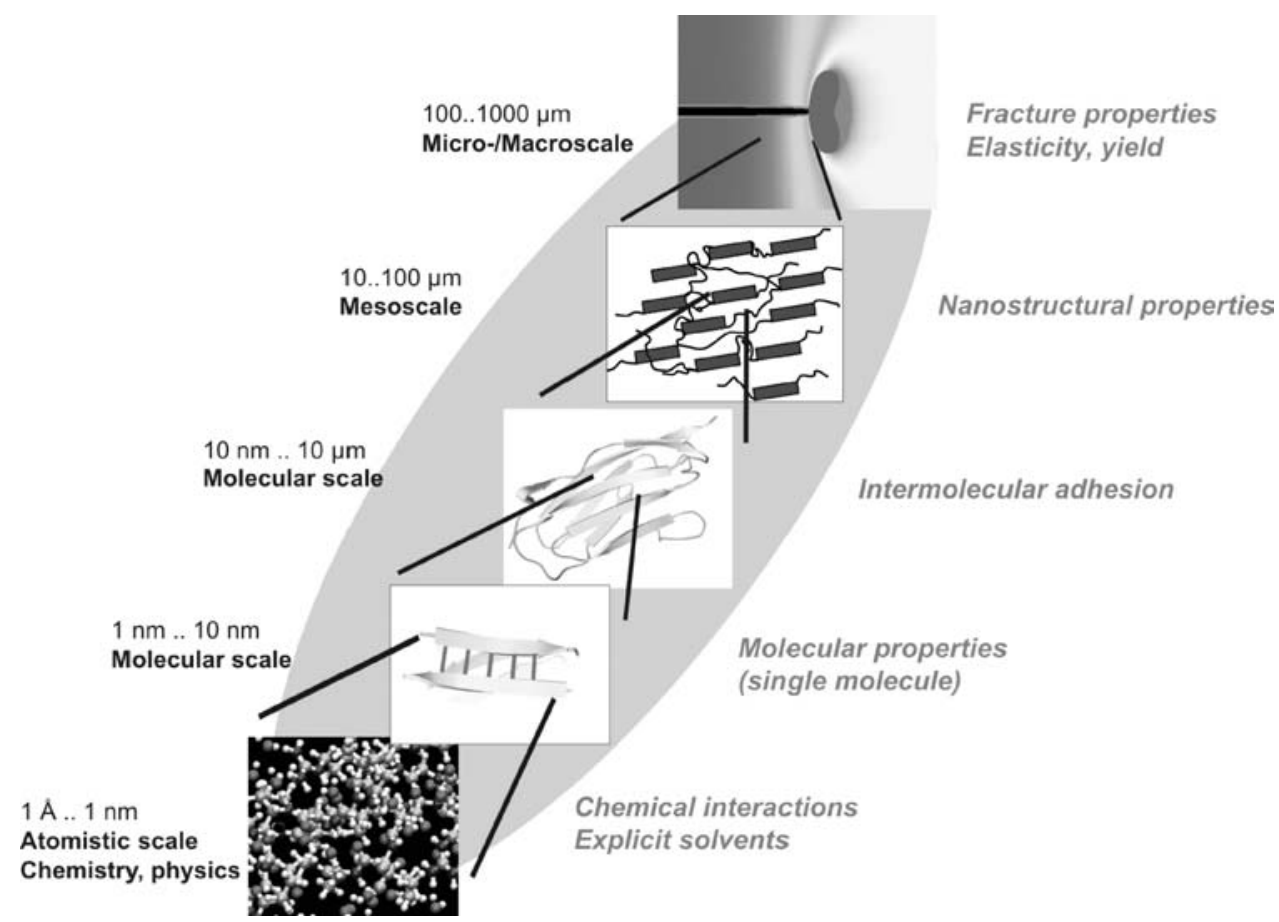

Figure 1. Overview over different material scales, from nano to macro (Buehler 2007). The macroscopic mechanical material behaviour is controlled by the interplay of properties throughout various scales. In order to understand deformation and fracture mechanisms, it is crucial to elucidate atomistic and molecular mechanisms at each scale, and to appreciate the cross-scale interaction of these mechanisms.

The mechanical properties of biological materials have wide ranging implications for biology. In cells for instance, mechanical sensation is used to transmit signals from the environment to the cell nucleus or to control tissue formation and regeneration (Alberts et al. 2002; Engler et al. 2006). The structural integrity and shape of cells is controlled by the cell's cytoskeleton, which resembles an interplay of complex protein structures and signaling cascades arranged in a hierarchical fashion (Alberts et al. 2002). Bone and collagen, providing structure to our body, or spider silk, used for prey procurement, are examples of materials that have incredible elasticity, strength and robustness unmatched by many synthetic materials, mainly attributed to its structural formation with molecular precision (Ramachandran and Kartha 1955; Currey 2002; Kitano 2002a, 2002b; An et al. 2004; Fratzl et al. 2004; Buehler 2006a, 2006b; Doyle 2007). The transfer of concepts observed in biology into technological applications and new materials design remains a big challenge that has, however, potential for huge payoff. In particular, the combination of nanostructural and hierarchical features into materials developments could lead to significant breakthroughs.

What are the most promising strategies in order to analyze these materials? Perhaps, an integrated approach that uses experiment and simulation concurrently could evolve into a new paradigm of materials research. Experimental techniques have gained unparalleled accuracy in both length- and time-scales (see Figure 2), as reflected in development and utilisation of atomic force microscope (AFM) (Prater et al. 1990; Smith et al. 1999), optical tweezers (Dao et al. 2003; Sun et al. 2004) or nanoindentation (Tai et al. 2006) to analyse biological materials (Lim et al. 2006).

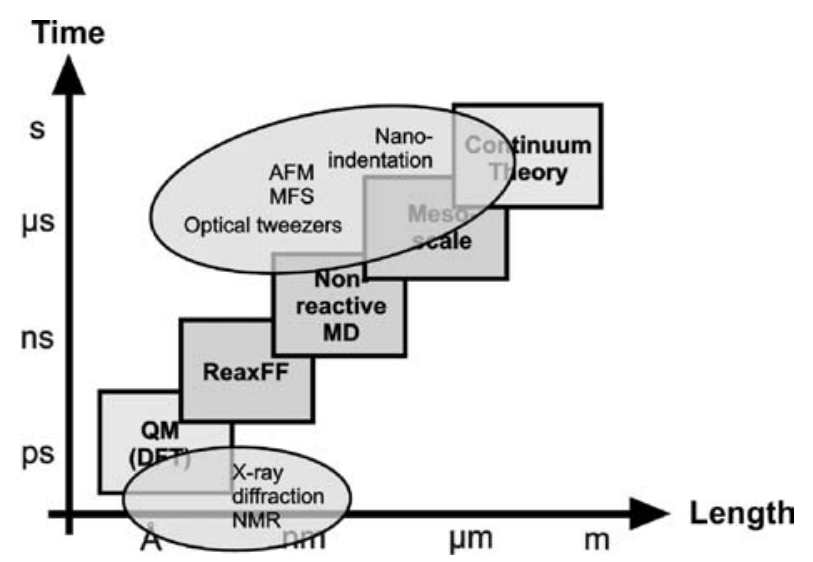

Figure 2. Overview over various computational and experimental tools (Buehler 2007). Hierarchical coupling of different computational tools can be used to traverse throughout a wide range of length- and time-scales. The handshaking between different methods enables one to transport information from one scale to another. The results of atomistic, molecular or mesoscale simulation may feed into constitutive equations or continuum models. Experimental techniques such as atomic force microscope (AFM), molecular force spectroscopy (MFS), nanoindentation or optical tweezers now overlap with atomistic and molecular approaches, enabling a direct comparison. 
Despite enormous progress in experimental methods, these methods are typically limited to particular spatial and temporal scales, compromising the ability to traverse through multiple scales with a single, consistent analysis method.

Predictive atomistic simulation is a promising approach to overcome these limitations. Atomistic simulation (Allen and Tildesley 1989) predicts the motion of all atoms in a biological system - including solvents by solving Newton's equations, $F=m a$, by incorporating appropriate empirical (Wang et al. 2001) or first principles based force fields (Duin et al. 2001; Buehler 2007) to model the chemical interactions.

Such predictive modelling and simulation have evolved into predictive tools that complement experimental analyses (see Figure 2). It is now feasible to start from smallest scale considering electrons and atoms, to reach all the way up to macroscopic scales of entire tissues (Goddard 2006), by explicitly considering the characteristic structural features at each scale. Even though there are still major challenges ahead of us, this progress is amazing and provides one with infinite possibilities and potentials, transforming materials science as a discipline through increased integration of computational approaches in scientific research. Methods such as the quasicontinuum approach or coarse-graining are important tools to enable such simulation studies.

\subsection{Cross-scale interactions}

A central theme of the efforts in developing the materials science of BPMs is to appreciate the structure-property or structure-processing-property paradigm. This is an important paradigm in the materials science community, which has guided materials research for many decades. For biological materials, there are many challenges that make developing these rigorous links more difficult (Buehler 2007).

For example, the bond energies of some bonds biological materials are often comparable to the thermal energy, as for instance in the case of hydrogen bonding, the most abundant chemical bond in biology. Many biological materials show viscoelastic behaviour, since their response to mechanical deformation is intrinsically time-dependent. In many cases, biological structures contain extremely compliant filaments, in which entropic contributions to the free energy are important and can even control the deformation behaviour. Many material properties are also length scale dependent and can vary significantly across various length scales. Quite often, this can be quite perplexing, since measuring different volumes of material lead to different values of Young's modulus. Size effects are often very strong and possibly utilized systematically to ensure physiological functioning of the material in its biological context. However, why and how these size effects are exploited within this context is often poorly understood (Buehler 2007). The presence of hierarchical structures calls for new paradigms in thinking (a)

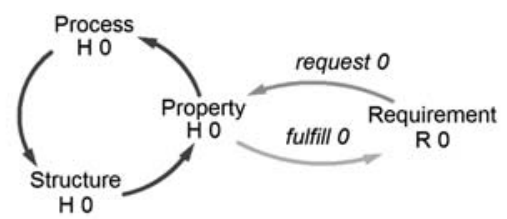

390

(b)

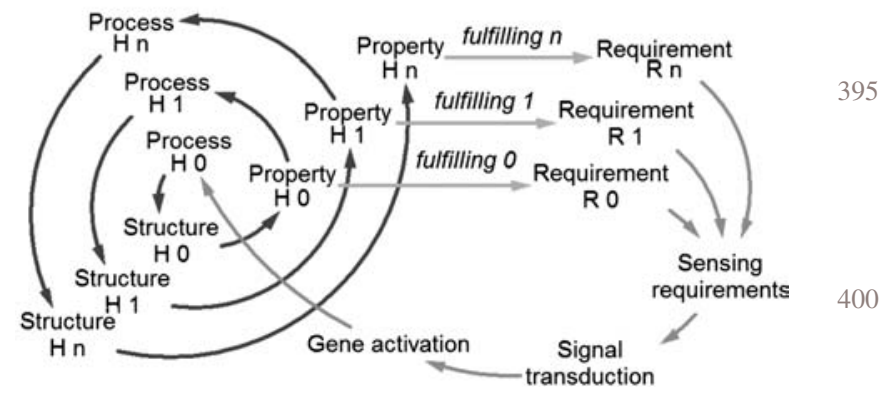

Figure 3. In BPMs, decentralised processes, material properties and environmental requirements, are brought together in mutual completion (Ackbarow and Buehler). Subplot (a) illustrates the traditional paradigm in materials science where process, structure and property build the 'magic' triangle on a single hierarchical level. Subplot (b) illustrates the paradigm for hierarchical (biological) materials. In contrast to the traditional paradigm, relations between 'external' functions or requirements and 'internal' properties exist on several scales resulting in multifunctionality. Further, as requirements consistently change overtime (e.g. changing loads, changing environment), a continuous adaptation is necessary. In addition to multi-functionality, robust feedback loops that result in smart signalling chains enable a decentralized self-organisation. Consequently, in PBMs hierarchy level specific properties $\left(H_{i}\right)$ do not only fulfill the required functions, but also initiate the decentralized processes on the next hierarchical level $\left(H_{i+1}\right)$, and thus generate the structures on this level $\left(H_{i+1}\right)$.

about the structure-property paradigm, since corresponding concepts must include an explicit notion of the crossscale and inter-scale interactions (Ackbarow and Buehler). Figure 3 illustrates how such a paradigm could be formulated for BPMs.

Examples for cross-scale interactions with biological and medical relevance include genetic diseases, injuries and healing processes. In genetic diseases (e.g. the collagen related disease osteogenesis imperfecta, the rapid ageing disease progeria that originates in changes of the lamin protein structure in the nuclear envelope, and many more), a core question is to understand the effect of modifications of the amino acid sequence on the behaviour of the biological system (e.g. the cell, the nuclear envelope). In injuries and healing processes, the effect of a macroscopic impact during an accident, for instance, causes damage across multiple scales, from macro to nano. The understanding of the details of the damage (e.g. molecular rupture, loss of protein structure, protein misalignment and others) due to the macroscopic impact is crucial to develop treatment strategies to facilitate and support healing processes. 440 
The finer scale knowledge is absolutely needed to explain phenomenological phenomena as well as to engineer a specific phenomenological behaviour. In addition, the signalling processes outlined in Section 1 represent examples for cross-scale phenomena, in which mechanical signals at the molecular scale influence cells or tissues.

The focus of this article is on deformation and rupture processes, that is, the loss of the equilibrium structure of protein building blocks. Another, very important area of research is the study of assembly of protein materials. For example the investigation of the protein-folding problem remains a computationally daunting task that can thus far only be carried out for relatively simple protein structures. Interesting cross-scale interactions are also expected to control these properties. In the future, the folding and assembly problem must be considered simultaneously with the study of rupture and deformation, the two represent opposing and competing mechanisms of synthesis and separation. A true understanding of biological protein materials must include these mechanisms in its description.

\subsection{Predictive strength models for hierarchical protein materials: Hierarchical Bell Model}

It has become evident that the atomistic scale, and in particular the notion of a chemical bond, provides a very fundamental, universal platform at which a variety of scientific disciplines can interact. Chemists, through the molecular structure of proteins, physicists, through the statistical mechanics of a large number of atoms, and materials scientists through analysis of phenomena such as elasticity, optical properties, electrical properties or thermodynamics, linking structure and function (Buehler, 2007). Hydrogen bonds (HBs) are a particularly important class of chemical bonds in BPMs. In many BPMs, hierarchical networks of HBs provide the thermodynamical and mechanical stability. Thus the understanding of fracture and deformation depends critically on the availability of models to capture the properties of HBs.

The key to comprehend the mechanical response of BPMs across different time- and length scales is to understand the rupture mechanics of HBs under laterally applied load. Typically, a variety of unfolding processes exist for a protein structure, each of which has a specific reaction pathway and an associated energy barrier, mostly related to the underlying bond breaking mechanisms and rearrangements in the protein structure. Several theories exist that describe competing processes due to mechanically induced instabilities of protein structures. These concepts stem primarily from a theory originally postulated by Bell (1978), Evans and Ritchie (1997), Gilli et al. (2004), Dudko et al. (2006) and Wiita et al. (2006).

Whereas continuum theories are suitable to describe the deformation of macroscopic structures, statistical concepts must be employed to accurately model the mechanical behaviour of protein structures. Bell's theory is one of the most widely used models to describe the statistical nature of bond breaking.

In Bell's theory (Bell 1978; Evans and Ritchie 1997; Gilli et al. 2004; Dudko et al. 2006; Wiita et al. 2006), the off rate $\chi$ is the product of the natural bond vibration frequency, $\omega_{0}$, and the quasi-equilibrium likelihood of reaching the transition state with an energy barrier $E_{\mathrm{b}}$ that is reduced by mechanical energy $F \cdot x_{\mathrm{b}} \cdot \cos (\theta)$, where $F$ is the applied force, $x_{\mathrm{b}}$ is the distance between the equilibrated state (minimum of the well) and the transition state, and $\theta$ is the angle between the direction of the reaction pathway of bond breaking ( $x$-direction) and the direction of applied load $F$ (see also the schematic shown in Figure 4). The angle can be determined by analysing the molecular geometry. The off rate describes how often a bond is broken per unit time (it equals to the reciprocal of the lifetime of a bond), and is given by

$$
\chi=\omega_{0} \cdot \exp \left(-\frac{\left(E_{\mathrm{b}}-F \cdot x_{\mathrm{b}} \cdot \cos (\theta)\right)}{k_{\mathrm{b}} \cdot T}\right) .
$$

The natural vibration frequency of a bond is $\omega_{0} \approx 1 \times 10^{13} \mathrm{~s}^{-1}$ (Bell 1978).

(a)

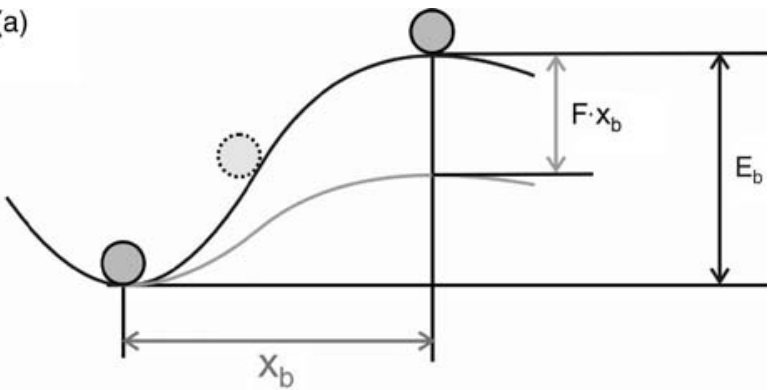

(b)

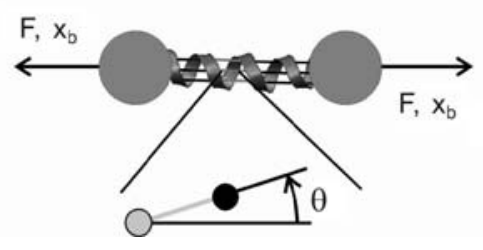

Figure 4. Statistical theory to predict the bond rupture mechanics (Ackbarow et al. 2007; Buehler and Ackbarow 2007). The graph depicts the energy as a function of deformation along a deformation variable (subplot (a)), along a particular pathway that leads to bond rupture; where $F$ is the applied force, and where $x_{\mathrm{b}}$ is the displacement in the direction of the applied force. In the schematic, three HBs [indicated by the red colour, subplot (b)] break simultaneously. Thus, $x_{\mathrm{b}}$ corresponds to the lateral displacement that is necessary to overcome the bond breaking distance of a $\mathrm{HB}$, in the particular atomistic geometry present in coiled-coil (CC) protein structures (Ackbarow and Buehler 2007). Given that $x_{\mathrm{b}}^{*}$ is the distance to break a single HB, the distance $x_{\mathrm{b}}^{*}=x_{\mathrm{b}} \cos \theta$ denotes the lateral displacement at bond breaking, with the angle $\theta$ as the angle between pulling direction and orientation of the HB inside the molecule. 
However, Equation (1) does not describe the dependence of the pulling speed (the controlled parameter in experiment and MD simulation) at which a bond breaks due to the applied pulling force. Instead, it only provides an estimate of the time scale at which the bond will be broken. In order to overcome this limitation, we modify Equation (1) based on the following idea: The speed $v$ at which a bond is broken equals to the distance that needs to be overcome in order to break the bond $\left(x_{\mathrm{b}}\right)$ divided by the time for the bond breaking. Consequently, $v$ is the product of $\chi \cdot x_{\mathrm{b}}$. This leads to the following equation for the bond breaking speed (Ackbarow and Buehler 2007):

$$
v=\omega_{0} \cdot x_{\mathrm{b}} \cdot \exp \left(-\frac{\left(E_{\mathrm{b}}-F \cdot x_{\mathrm{b}} \cdot \cos (\theta)\right)}{k_{\mathrm{b}} \cdot T}\right) .
$$

This equation can be rewritten in the following way:

$$
v=v_{0} \cdot \exp \left(\frac{F \cdot x_{\mathrm{b}} \cdot \cos (\theta)}{k_{\mathrm{b}} \cdot T}\right)
$$

with $v_{0}$ as the as natural bond breaking speed (when no load is applied), defined as:

$$
v_{0}=\omega_{0} \cdot x_{\mathrm{b}} \cdot \exp \left(-\frac{E_{\mathrm{b}}}{k_{\mathrm{b}} \cdot T}\right) .
$$

This modified framework enables us to study the dependence between the unfolding force and the bond breaking speed or to calculate the average force at which a Equation (3) as:

$$
F(v)=\frac{k_{\mathrm{b}} \cdot T}{x_{\mathrm{b}} \cdot \cos (\theta)} \cdot \ln v-\frac{k_{\mathrm{b}} \cdot T}{x_{\mathrm{b}} \cdot \cos (\theta)} \cdot \ln v_{0}=a \cdot \ln v+\mathrm{b},
$$

where $a=k_{\mathrm{b}} \cdot T /\left(x_{\mathrm{b}} \cdot \cos \theta\right)$ and $b=-k_{\mathrm{b}} \cdot T /\left(x_{\mathrm{b}} \cdot \cos \theta\right) \cdot \ln v_{0}$. Equation (5) predicts that the unfolding force depends logarithmically on the pulling speed in a non-equilibrated system. We note that it contains two parameters $a$ and $b$, which can be calculated from the parameters $x_{\mathrm{b}}$ and $E_{\mathrm{b}}$ at a certain temperature and angle. The concept is schematically summarized in Figure 4.

Even though the phenomenological model (Equation (5) and previous equations) explicitly considers chemical 'bonds', it does not distinguish between a single chemical bond and protein architectures that include several bonds. For instance, whether a single HB ruptures or if several HBs rupture simultaneously is captured in an effective value of $E_{\mathrm{b}}$; however, this change in mechanism is not explicitly noted in the theory (Ackbarow et al. 2007; Ackbarow and Buehler).

In order to predict the strength and the energy landscape of a protein without performing simulations or experiments, we extend the theory to explicitly consider the structural hierarchies of the protein structure with the bond breaks, at a certain pulling rate. We can rewrite only input parameters being the energy of a $\mathrm{HB}$ and the rupture distance. The $\mathrm{AH}$ represents a hierarchical structure, ranging from individual HBs at the lowest, atomistic level to a collection of HBs at the next higher, molecular protein scale.

The lowest hierarchy is represented by individual HBs with an $E_{\mathrm{b}}^{0}$ and $x_{\mathrm{b}}$, and the higher hierarchy consists of parallel HBs. Here, we assume that $b$ bonds in a structure are in parallel and $d$ bonds out of these $b$ bonds break simultaneously. Thus ${ }_{\mathrm{b}} C_{\mathrm{d}}$ possible combinations exist for this rupture mechanism (the binomial coefficient is defined as $\left.{ }_{\mathrm{b}} C_{\mathrm{d}}=\left(\begin{array}{l}b \\ d\end{array}\right)=b ! /(b-d) ! / d !\right)$. The probability that one of these combinations constitutes a particular rupture event is one divided by ${ }_{\mathrm{b}} C_{\mathrm{d}}$. Also, if $d$ bonds break simultaneously, the total energy barrier increases by a factor $d$, to $d \cdot E_{\mathrm{B}}^{0}$. This leads to the following expression for the off rate:

$$
\chi_{\mathrm{H}}=\omega_{0} \cdot\left(\begin{array}{l}
b \\
d
\end{array}\right)^{-1} \cdot \exp \left(-\frac{\left(d \cdot E_{\mathrm{b}}^{0}-F \cdot x_{\mathrm{b}} \cdot \cos (\theta)\right)}{k_{\mathrm{B}} \cdot T}\right) .
$$

We rewrite Equation (6) so that the binomial coefficient appears in the exponential, which enables us to compare Equation (6) with Equation (1),

$\chi_{\mathrm{H}}=\omega_{0} \cdot \exp \left(-\frac{\left(d \cdot E_{\mathrm{b}}^{0}+k_{\mathrm{B}} \cdot T \cdot \ln \left(\begin{array}{l}b \\ d\end{array}\right)-F \cdot x_{\mathrm{b}} \cdot \cos (\theta)\right)}{k_{\mathrm{B}} \cdot T}\right)$.

The parameter $E_{\mathrm{b}}$ can thus be split up as

$$
E_{\mathrm{b}}=d \cdot E_{\mathrm{b}}^{0}+k_{\mathrm{b}} \cdot T \cdot \ln \left(\begin{array}{l}
b \\
d
\end{array}\right)
$$

where $E_{\mathrm{b}}^{0}$ is the energy of a single bond and the term $k_{\mathrm{b}} \cdot T \cdot \ln \left(\begin{array}{l}b \\ d\end{array}\right)$ is the contribution to the energy barrier due to the hierarchical structure. The unfolding force is

$$
\begin{aligned}
F\left(v, b, d ; E_{b}^{0}, x_{\mathrm{b}}, \theta\right)= & \frac{k_{\mathrm{B}} \cdot T}{x_{\mathrm{b}} \cdot \cos \theta} \ln \left(\frac{v}{x_{\mathrm{b}} \cdot \omega_{0}}\right) \\
& +\frac{k_{\mathrm{b}} \cdot T}{x_{\mathrm{b}} \cdot \cos \theta} \cdot \ln \left(\begin{array}{l}
b \\
d
\end{array}\right)+\frac{d \cdot E_{\mathrm{b}}^{0}}{x_{\mathrm{b}} \cdot \cos \theta} .
\end{aligned}
$$

Note that $F=F_{v}+F_{h 1}+F_{h 0}$, where the $F_{v}, F_{h 1}$ and $F_{h 0}$ are the contributions to the force as a consequence 
of the pulling speed, the first hierarchy (number of parallel bonds, $d$ ), and the basic hierarchy (strength of bonds, $E_{\mathrm{b}}$ and $x_{\mathrm{b}}$ ). This expression quantifies how the hierarchical design influences the rupture strength. In the following, we refer to this model as the Hierarchical Bell model, as proposed originally in Ackbarow et al. (2007).

We note that this approach can easily be extended to three hierarchies, which enables one to predict the rupture force of a tertiary structure consisting of $2,3, \ldots n \mathrm{AHs}$, of which $k$ unfold simultaneously (Ackbarow et al. 2007).

We emphasize the significance of the expression given in Equation (9): In contrast to the phenomenological description of the energy barrier in the conventional Bell Theory (Equations (1) to (5)) that cannot distinguish between single HB breaking or multiple HB breaking, the expression in Equation (9) explicitly considers the effect of the hierarchical structure, and the fit of experimental or simulation results to this model provides a direct measure of the energy of a single HB. We note that different variables are used to express this effect: $E_{\mathrm{b}}^{0}$ refers to the energy of a single $\mathrm{HB}$, whereas $E_{\mathrm{b}}$ represents the energy barrier of an assembly of HBs.

\subsection{Simulation approach}

We use a classical molecular dynamics (MD) approach, implemented in NAMD (Nelson et al. 1996) using the CHARMM22 force field (MacKerell et al. 1998). All simulations were performed at a temperature of $300 \mathrm{~K}$ ( $N V T$ ensemble, Berendsen thermostat), with a time step 1 fs. Careful energy minimisation and finite temperature equilibration of all structures are achieved before the protein is loaded. The structure obtained from the Protein Data Bank (PDB) is solved completely in a TIP3 water skin (TIP3 represents a molecular force field model for water molecules and their interactions). The entire protein is embedded in water, before and during deformation of the protein. The total system has approximately 10,000 atoms in a non-periodic simulation, with the total simulation time exceeding several months for the longest simulations that reach several hundred nanoseconds. This illustrates some of the limitations of the atomistic approach, partly due to the extreme computational requirements. The inclusion of explicit water molecules is essential to capture the correct HB rupture dynamics that may be strongly influenced by the water molecules.

To apply forces to the molecule in order to induce deformation, we use steered molecular dynamics (SMD) (Lu et al. 1998), with SMD spring constant of $k_{\mathrm{SMD}}=$ $10 \mathrm{kcal} / \mathrm{mol} / \AA^{2}$. We obtain force-versus-displacement data by monitoring the time-averaged applied force $(F)$ and the position of the atom that is pulled at $(x)$ over the simulation time.

The $\mathrm{AH}$ atomistic structure is taken from the $2 \mathrm{~B}$ segment of the vimentin intermediate filaments (IF) (Wang and Stamenovic 2002; Helfand et al. 2004; Mucke et al. 2004) coiled-coil dimer, length $70 \AA$ (PDB identification 1gk6; '2D' refers to a particular domain of the larger protein). For this molecular structure, the angle $\theta \approx 16^{\circ}$.

\subsection{Computational results}

For the vimentin $\mathrm{AH}$ protein domain, two characteristic force-strain curves are shown in Figure 5 for two pulling speeds (Ackbarow et al. 2007). The simulations reveal the existence of three distinct deformation regimes. The first regime shows a linear increase in strain, followed by
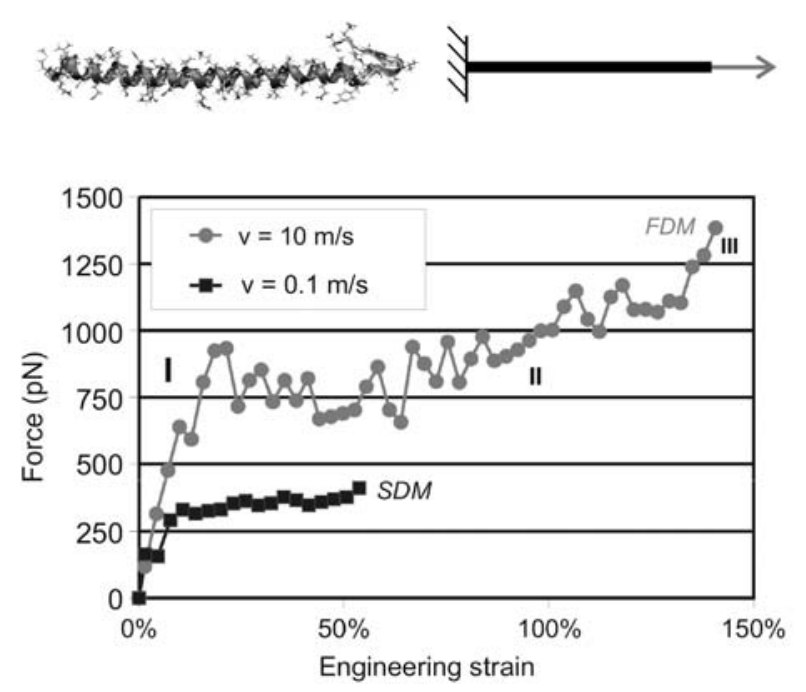

Figure 5. Upper part: atomistic geometry of the AH molecule studied (explicit water solvent not shown for clarity), and boundary condition for the pulling experiment. Lower part: examples for force-extension curves, as reported in Ackbarow et al. (2007). The fast deformation mode (FDM) is represented by a curve taken at a pulling speed of $10 \mathrm{~m} / \mathrm{s}$. The slow deformation mode $(\mathrm{SDM})$ is represented by a pulling experiment at $0.1 \mathrm{~m} / \mathrm{s}$. The force-extension behaviour consists of three regimes: (I) linear increase in strain until the angular point is reached when the first HBs rupture, leading to unfolding of one helical convolution. (II) Plateau of approximately constant force, during which unfolding of the entire protein occurs. (III) Strain hardening (only partly shown for the FDM). We refer the reader to the literature for an analysis of the large-deformation regime of the FDM (Ackbarow and Buehler 2007). 
a plateau indicating the second stage. This second regime appears at approximately constant force, during which unfolding of the entire protein occurs. The last regime displays a significant strain hardening, due to pulling of the protein's backbone (only partly visible in the FDM plot). The change from the first to the second regime is referred as the angular point (AP), denoting the protein unfolding force. Unfolding of the protein is characterized by rupture of HBs that destroy the protein structure as the displacement is increased. In the remainder of this article, we focus on the force at the AP as a function of the pulling speed.

We now carry out computational experiments by systematically varying the pulling velocity over four orders of magnitude, ranging from $0.05 \mathrm{~m} / \mathrm{s}$ to $100 \mathrm{~m} / \mathrm{s}$. The unfolding force is plotted as a function of the pulling speed in Figure 6. Notably, we observe two distinct regimes, each of which follows a logarithmic dependence of the unfolding force with respect to the pulling rate. The existence of two discrete slopes indicates two different energy barriers and thus two different unfolding mechanisms over the simulated pulling velocity regime. The results clearly suggest a free energy landscape that consists of two transition states, that is, two pairs of $E_{\mathrm{b}}$ and $x_{\mathrm{b}}$. In the following, we refer to these two regimes as the slow deformation mode (SDM) and the fast deformation mode (FDM). The change in mechanism from the FDM the SDM occurs at $v=0.4 \mathrm{~m} / \mathrm{s}$, at a force of $\approx 350 \mathrm{pN}$.

We emphasize that the change in mechanism has thus far only been suggested or inferred (Gao et al. 2002; Sotomayor and Schulten 2007). For example, a comparison between MD simulation and experimental results

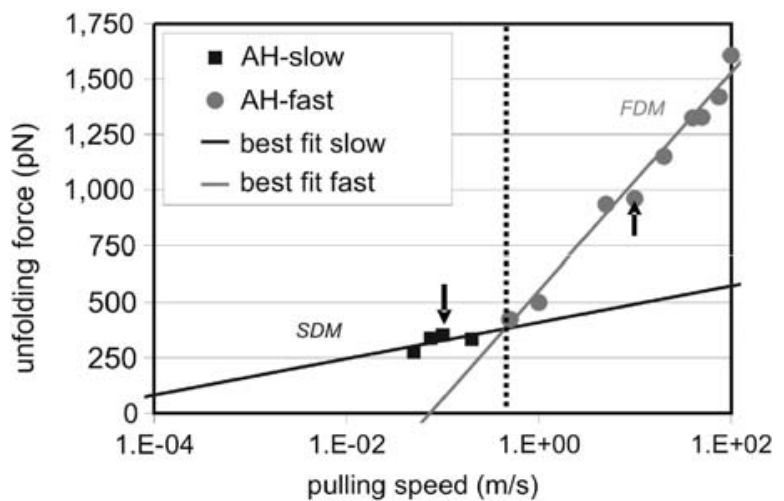

Figure 6. Unfolding force of single AHs from the vimentin coiled-coil dimers, as a function of varying pulling speed over four orders of magnitude, ranging from 0.05 to $100 \mathrm{~m} / \mathrm{s}$, as reported in Ackbarow et al. (2007). The arrows indicate the representative pulling speeds used for the analysis reported in the other figures. As predicted by Equation (5), the unfolding force of the AH depends logarithmically on the pulling speed. The results clearly reveal a change in mechanism from fast deformation mode (FDM) to the slow deformation mode (SDM) at $0.4 \mathrm{~m} / \mathrm{s}$ pulling speed, and at a force of approximately $350 \mathrm{pN}$. This suggests a free energy landscape that consists of two transition states for the regime studied here. revealed that force-pulling speed dependence must lay on two different curves in the $f-\ln (v)$ plane (Gao et al. 2002; Sotomayor and Schulten 2007), suggesting a change in unfolding mechanism. To the best of our knowledge, the atomistic simulation results shown in Figure 6 are the first direct confirmation of this phenomenon. This illustrates the significance of our findings.

Further evidence for the change in mechanism is obtained by an analysis of the HB rupture dynamics. In Figure 7 we plot the $\mathrm{HB}$ rupture as a function of the molecular strain for the vimentin AH1 domain. This provides a strategy to normalize the different time scales by the pulling velocity (here 0.1 and $10 \mathrm{~m} / \mathrm{s}$ ). In agreement with the results shown in Figure 6, the unfolding of the protein in the SDM starts at approximately $10 \%$ strain, in contrast to $20 \%$ strain in the FDM regime. This is indicated by the rupture of the first HB. The data shown in Figure 7 clearly suggests that in the FDM, HBs rupture sequentially as the lateral load is increased from 20 to $40 \%$ tensile strain. In contrast, in the SDM several HBs rupture virtually simultaneously, within less than $20 \mathrm{ps}$, at a tensile strain of $\approx 10 \%$. Even though the pulling speed is several orders of magnitude slower in the SDM, the HBs in the SDM rupture significantly faster. The physical reason for this observation may be that HB rupture in the FDM is controlled by increases in the applied strain, and thus depends on the strain rate. On the other hand, in the SDM,

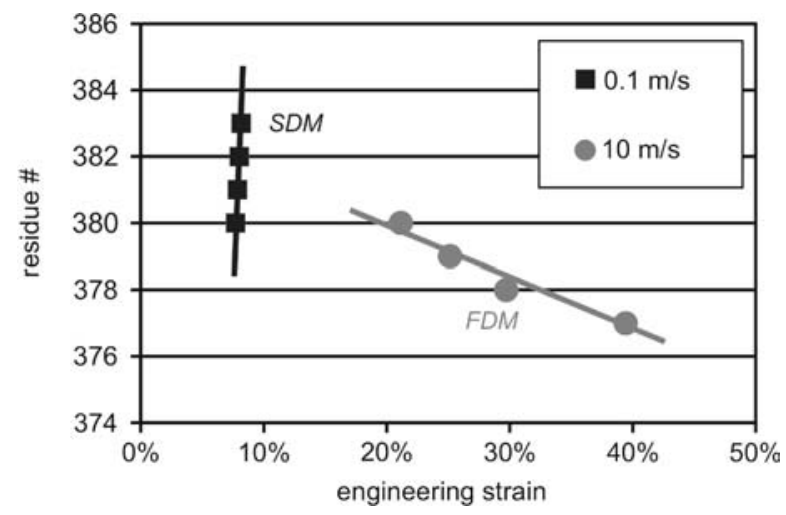

Figure 7. The rupture sequence of the first four HBs (approximately one convolution), as a function of the applied strain, as reported in Ackbarow et al. (2007) (the sequence of rupture was determined by analysing the time sequence of rupture along with the geometric position of the HBs). The residue number represents the amino acid of the O-atom (hydrogen acceptor). In the FDM the HBs rupture one by one, whereas in the SDM several HB rupture virtually simultaneously, within 20 ps. This can be quantified by the HB breaking 'speed', the slope of the best linear fit in this graph. The speed is more than 40 times faster in the SDM $(\approx 7 \mathrm{HBs}$ break per $1 \%$ strain $)$ than in the FDM (0.16 HBs per $1 \%$ strain). Even though the pulling speed is 100 times slower and thus the time scale is 100 times smaller, the HBs in the SDM rupture significantly faster. The unfolding in the SDM regime begins at $10 \%$ strain in contrast to the $20 \%$ strain in the FDM. We currently have no explanation for the difference in the slope in the two cases. 
।

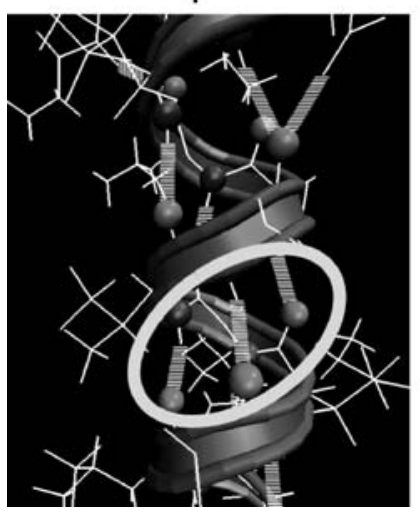

II

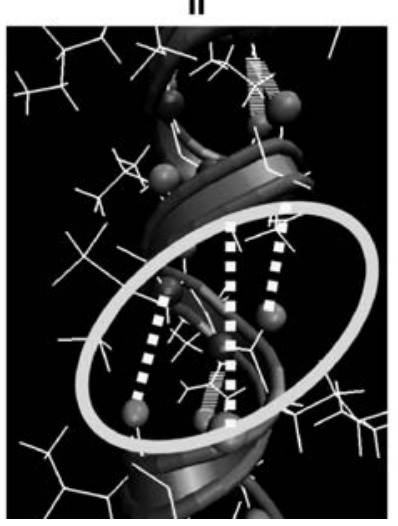

III

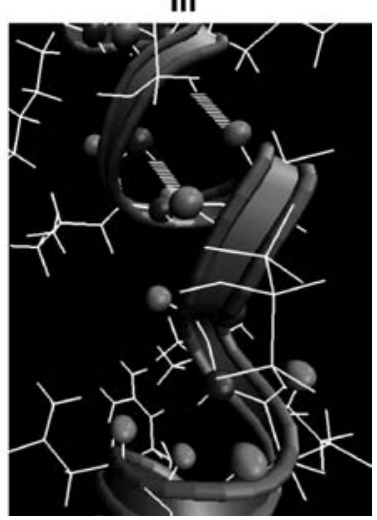

940

Figure 8. The unfolding of the AH in the SDM (angular point in Figure 5 for $v=0.1 \mathrm{~m} / \mathrm{s}$ ) starts with simultaneous rupture of three HBs. The time interval between these snapshots is 20 ps (between I and II) and 40 ps (between II and III). After 20 ps, all three HBs rupture simultaneously, leading to local unfolding of the protein in the following $40 \mathrm{ps}$ (from II to III). It was reported in the literature that the time for HB breaking is approximately $20-40$ ps (Sheu et al. 2003). Thus, these snapshots strongly support the concept of cooperative bond rupture in the SDM. Surrounding water molecules are not shown for clarity.

the cluster of HBs ruptures within a few tens of picoseconds, regardless of strain rate (this time scale of $\mathrm{HB}$ rupture is an intrinsic property of HBs). Since clusters of 3-4 HBs rupture simultaneously, rupture proceeds at a much faster rate.

A detailed analysis of the atomistic structure during HB rupture is shown in Figure 8 for the SDM. This analysis provides additional proof that in the SDM three HBs rupture simultaneously, within less than $20 \mathrm{ps}$ time scale. We note that it was reported in the literature that the time for HB breaking is of approximately $20-40$ ps (Sheu et al. 2003), clearly supporting the notion that these HBs rupture simultaneously.

By fitting the Extended Bell Theory to the MD results we can obtain quantitative estimates of the rupture forces. In the $\operatorname{SDM}$ (with $\theta \approx 16^{\circ}$, as obtained from the molecular geometry), $E_{\mathrm{b}}=11.1 \mathrm{kcal} / \mathrm{mol}$ and $x_{\mathrm{b}}=1.2 \AA$. Since, three HBs break simultaneously, $E_{\mathrm{b}}^{0}=3.7 \mathrm{kcal} / \mathrm{mol}$, with $b=3$ and $d=3$. Further, we obtain for the FDM $E_{\mathrm{b}}=4.7$ $\mathrm{kcal} / \mathrm{mol}$ and $x_{\mathrm{b}}=0.20 \AA$, which leads to $E_{\mathrm{b}}^{0}=$ $4.1 \mathrm{kcal} / \mathrm{mol}$ with $b=3$ and $d=1$. Notably, the estimated values of $E_{\mathrm{b}}^{0}$ are similar in both cases (3.7 versus $4.1 \mathrm{kcal} / \mathrm{mol}$ ). The slight discrepancy may be due to the fact that the mechanisms in the SDM and FDM are slightly different that assumed in our model. However, overall the estimate provided from this model is a reasonable approximation. The reason for the difference of the values of $x_{\mathrm{b}}$ between the SDM and FDM is likely the fact that a different energy pathway is activated. In particular, in the FDM the molecular strain is not equilibrated, which leads to a localised unfolding, similar to a stress concentration in solids. In this sense, the local displacements are larger than the applied displacements, which lead to a muchreduced value for the transition point. We note that $x_{\mathrm{b}}^{*}$ (the local transition point for rupture of a H-bond) is always the same in both FDM and SDM. However, the value of $x_{\mathrm{b}}$, the measured transition point, due to application of global load at the ends of the molecule, changes (see also Figure 4).

We note that the bond breaking energy $E_{\mathrm{b}}^{0}$ of a HB in water ranges from 3 to $6 \mathrm{kcal} / \mathrm{mol}$ (Sheu et al. 2003), providing reasonable agreement with the computational results.

\subsection{Comparison with experimental results}

Notably, experimental results for single AH experiments are very rare, even though the first experiments have been reported almost 10 years ago. The forces measured in unfolding experiments are typically between 100 and 200 pN (Lantz et al. 1999; Mitsui et al. 2000; Kageshima et al. 2001). However, experimentalists have reported difficulties due to the small size of the protein probes, and the lack of control to stretch individual AHs rather than bundles (Lantz et al. 1999; Mitsui et al. 2000; Kageshima et al. 2001).

In light of these difficulties of stretching single AHs, more relevant results are those derived from pulling experiments of coiled-coils, which consist of two AHs arranged in a helical geometry. In these systems, the unfolding force measured in experiment ranges between 25 and $110 \mathrm{pN}$, for pulling velocities between $8 \mathrm{E}-8$ and $5 \mathrm{E}-7 \mathrm{~m} / \mathrm{s}$ (Schwaiger et al. 2002; Kiss et al. 2006). These force values are in proximity to the unfolding forces predicted by our simulations and theoretical model (extrapolated values based on the behaviour in the SDM). The observation of the change of mechanism from the FDM to the SDM regime enables us to actually extrapolate estimates of the unfolding forces from MD simulation speeds, approaching the pulling speeds used in experiment [the predicted unfolding forces at $1 \mathrm{E}-7 \mathrm{~m} / \mathrm{s}$ are approximately $100 \mathrm{pN}$ (Ackbarow and Buehler 2007)]. 


\subsection{Summary}

By using an integrated approach of theory and simulation, we have systematically varied the pulling velocity and discovered a change in unfolding behaviour during stretching of $\mathrm{AH}$ protein domains. The results of the MD simulations were analysed using the Extended Bell Model, which enables us to calculate energy barrier associated with breaking individual HBs. The results suggest that the unfolding mechanism at fast pulling rates is rupture of a single $\mathrm{HB}$, whereas the unfolding mechanisms at slow pulling rates proceeds by simultaneous rupture of several parallel HBs (Ackbarow et al. 2007).

It is apparent that as of today, numerical MD simulations are the only means to directly observe these mechanisms, since experiments are still lacking appropriate spatial and temporal resolution. Advances in computing power has enabled us to carry out direct atomistic simulation of unfolding phenomena, including explicit solvent, at time scales approaching a significant fraction of a microsecond.

In previous atomistic simulations, unfolding forces have been significantly larger than those measured in experiment, likely because they were carried out in the FDM so that forces reach up to several $\mathrm{nN}$ for individual AHs. This is clearly an artifact of large pulling speeds, as pointed out earlier ( $\mathrm{Lu}$ and Schulten 1999; Sotomayor and Schulten 2007). Our analysis shows that in addition to incorrect force estimates, the observed unfolding mechanism can also be significantly different if the pulling speed is too high. The estimate for $v_{\text {cr }}$ provides a maximum pulling rate that could be used in MD studies, in order to still allow a reasonable interpretation of MD results in light of biological relevance. The quantitative value reported here may thus provide guidance to set up other MD simulations.

We note that the SDM is most relevant for biological function. However, the FDM could be important during tissue injuries that may be incurred under large deformation rates (e.g. shock impact, bullets and fractures).

\section{Q1 3. Future directions, challenges and impact}

Over the last centuries, engineers have developed understanding of how to create complex man-made structures out of a diverse range of constituents, at various scales (machines, buildings, airplanes, nuclear reactors and many others). Increased development and research funding into these areas of research will lead to breakthroughs not only on the fundamental sciences, but also in technological applications. Research in the area of mechanics of biological materials will extend our ability to carry out structural engineering, as used for buildings or bridges today, to the ultimate scale - nanoscale, and may be a vital component of the realisation of nanotechnology (Ackbarow and Buehler).
It is vital to overcome the barrier that currently separates the scales, through development of new methods, better model systems and an advanced appreciation for a multiscale view, in order to fully understand multi-scale or crossscale interactions. To facilitate these developments, we must also develop a proper nomenclature to capture the various scales involved in a material (Buehler 2007). Current terminologies referring to atomistic, meso, micro and macro are insufficient to capture the subtleties of the various scales. Research should address the question, what are the opportunities in integrating nanoscience and nanotechnology into biological research? What will and can our impact be, in a long perspective, in understanding fundamental biology? For instance, is the nanomechanics of protein materials significant for biology, and have biologists missed out on important effects due to lack of consideration of the nanomechanics? How does Nature synthesize materials that are environmentally friendly, lightweight and yet tough and robust and can serve multiple objectives? How is robustness achieved? How do universality and diversity integrate into biological structures?

From a theoretical viewpoint, major challenges are the development of new materials theories that include atomistic and statistical effects into an effective description, while retaining a system theoretical perspective (Bell 1978; Lakes 1993; Bustamante et al. 1994), maybe eventually leading to a merger between system biology and materials science (Ackbarow and Buehler).

Similar to dislocation mechanics for metal plasticity, what is the theoretical framework for the behaviour of biological materials and structures? It is possible that statistical theories may evolve into the theoretical language of nanomechanics (Bell 1978). Atomistic simulations of complex protein structures with explicit solvents are often prohibitive, and coarse-graining techniques are often used. However, how effective are coarse-graining techniques? Can we indeed average out over atomistic or mesoscale structures? How important are atomistic features at macroscale? What are the best numerical strategies to simulate the role of water in very small confinement? How does confined water influence the mechanics of natural and biological materials?

Progress in these various challenging fields will probably occur specific to problems and applications, perhaps in those have most impact in medical or economic fields. Eventually, we must generalise our insight into the formulation of a holistic theory that extends the current nomenclature, theory and experimental thinking. These efforts will provide the scientific and engineering fundamentals to develop and maintain the infrastructures to enable and evolve modern civilisation. Materials - and materials science - will likely play a seminal role in these developments.

A better understanding of the mechanics of biological and natural materials, integrated within complex technological systems will make it possible to combine living and
1050 
non-living environments to develop sustainable technologies. New materials technologies such as protein-based materials produced by recombinant DNA techniques represent new frontiers in materials design and synthesis (Langer and Tirrell 2004; Zhao and Zhang 2007). These questions have high impact in the understanding and the design of environmentally friendly technologies and may enhance the quality of life of millions of people, through advances in the medical sciences as well as through improvements of the living environment. Currently, a pressing question is the development of new technologies to address the energy problem. Advances may be possible by utilisation of bacteria to produce and process fuel from crops, or by enabling the synthesis of materials at reduced processing temperature.

Nanoscience and nanotechnology enable us to make structures at the ultimate scale (self-assembly, recombinant DNA, utilisation of motor proteins for nano-machines and many others). This will perhaps lead to novel complex structural materials, designed from nano to macro. The theoretical progress in understanding hierarchical biological materials will facilitate to use an extended physical space, through the use of multiple hierarchies, in an efficient and controlled manner, that is, lead to a bottom-up structural design on the sub-macroscopic scale, instead of trial-anderror approaches. For example, the extended design space might serve as a means to realise new physical realities that are not accessible to a single scale, such as material synthesis at moderate temperatures, or fault tolerant hierarchical assembly pathways (Holland 1995), which enable biological systems to overcome the limitations to particular chemical bonds (soft) and chemical elements (organic) present under natural conditions (Ackbarow and Buehler).

The improved understanding of the hierarchical design

1135 laws might further enable the development and application of new organic and organic-inorganic multi-featured composites (such as assemblies of carbon nanotubes and proteins or polymer-protein composites [Cui et al. 2007; Hule and Pochan 2007; Winey and Vaia 2007)], which will mainly consist of chemical elements that appear in our environment in an almost unlimited amount $(\mathrm{C}, \mathrm{H}, \mathrm{N}, \mathrm{O}$ and $\mathrm{S}$ ). These materials might consequently help to solve human's energy and resource problems (e.g. fossil resources, iron, etc.), and allow us to manufacture nanomaterials, which will be produced in the future by techniques like recombinant DNA (Smeenk et al. 1971; Petka et al. 1998; Langer and Tirrell 2004) or peptide selfassembly (Mershin et al. 2005; Zhao and Zhang 2006; Zhao and Zhang 2007), techniques where the borders between materials, structures and machines vanish.

Applications of these new materials and structures are new biomaterials, new polymers, new composites, engineered spider silk, new scaffolding tissues, improved understanding of cell-ECM interactions, cell mechanics, hierarchical structures and self-assembly. In addition to the long-term impact in biology, bioengineering and medicine, this research may eventually contribute to our theoretical understanding of how structural features at different scales interact with one another. In light of the 'extended physical design space' discussed above, this may transform engineering approaches not only for materials applications, but also in manufacturing, transportation or designs of networks.

The use of modelling and simulation in the study of biological protein materials is a promising avenue of research. However, atomistic simulation methods face many challenges. For instance, accessible time scales are typically limited to several hundreds of nanoseconds. The size of atomistic systems cannot exceed billions of particles; however, realistic biological systems feature $10^{23}$ and many more atoms. The definition of proper interatomic force fields and potentials is another grand challenge in this field. Methods to include more details of chemical complexity is crucial for the advancement of the modelling capabilities. We believe that the impact of modelling and simulation is particular meaningful in the context of an integration with experimental approaches. This provides validation and guidance for new model development, the design of new experiments, and ideas and concepts for new materials architectures.

The insights into important scaling laws of protein behaviour may also aid us in understanding why particular protein structures have evolved into particularly common motifs, such as $\alpha$-helices or $\beta$-sheets. For example, a characteristic dimension of $\mathrm{H}$-bond assemblies associated with a particularly high mechanical strength has recently been linked with the biological prevalence of the size of $\mathrm{H}$ bond clusters (Keten and Buehler 2008).

\section{Acknowledgements}

This research was supported by the Army Research Office (ARO), Grant No. W911NF-06-1-0291 (program officer Dr. Bruce LaMattina), the Solomon Buchsbaum AT\&T Research Fund, as well as a National Science Foundation CAREER Award CMMI-0642545 (program officer Dr. Jimmy Hsia). T.A. acknowledges the support of the German National Academic Foundation and the Dr.-Juergen-Ulderup Foundation.

\section{References}

Ackbarow T, Buehler MJ. Hierarchical coexistence of universality and diversity controls robustness and multifunctionality in protein materials. Theor. Comput. Nanosci.

Q2 in press.

Ackbarow T, Buehler MJ. Hierarchical coexistence of universality and diversity controls robustness and multi-function-

Q3 ality in protein materials, to be submitted

Ackbarow T, Buehler MJ. Molecular mechanics of stutter defects in vimentin intermediate filaments. Exp. Mech. in submission. 
Ackbarow T, Buehler MJ. 2007. Superelasticity, energy dissipation and strain hardening of vimentin coiled-coil intermediate filaments: Atomistic and continuum studies. J. Materi. Sci. 42(21):8771-8787.

Ackbarow T et al., 2007. Hierarchies, multiple energy barriers and robustness govern the fracture mechanics of $\alpha$-helical and $\beta$-sheet protein domains. Proc. Natl. Acad. Sci. USA. 104:16410-16415.

Aizenberg J et al., 2005. Skeleton of Euplectella sp.: Structural hierarchy from the nanoscale to the macroscale. Science 309(5732):275-278.

\section{Q4 Francis.}

Allen MP, Tildesley DJ. 1989. Computer simulation of liquids. Oxford: Oxford University Press.

An KN, Sun YL, Luo ZP. 2004. Flexibility of type I collagen and mechanical property of connective tissue. Biorheology 41(3-4):239-246.

Arzt E, Gorb S, Spolenak R. 2003. From micro to nano contacts in biological attachment devices. Proc. Natl. Acad. Sci. USA. 100(19):10603-10606.

Bell GI. 1978. Models for specific adhesion of cells to cells. Science 200(4342):618-627.

Blanckenhagen Bv, Gumbsch P, Arzt E. 2001. Dislocation sources in discrete dislocation simulations of thin film plasticity and the Hall-Petch relation. Model. Simul. Mater. Sci. Eng. 9:157-169.

Buehler MJ. 1961. Atomistic and continuum modeling of mechanical properties of collagen: Elasticity, fracture, and self-assembly. J. Mater. Res. 21(8):1947-1961.

Buehler MJ. 2006. Large-scale hierarchical molecular modeling of nanostructured biological materials. J. Comput. Theor. Nanosci. 3(5):603-623.

Buehler MJ. 2006. Nature designs tough collagen: Explaining the nanostructure of collagen fibrils. Proc. Natl. Acad. Sci. USA. 103(33): $12285-12290$.

Buehler MJ. 2006a. Atomistic and continuum modeling of mechanical properties of collagen: elasticity, fracture and self-assembly. J. Mater. Res. 21(8):1947-1961.

Buehler MJ. 2006b. Nature designs tough collagen: Explaining the nanostructure of collagen fibrils. Proc. Natl. Acad. Sci. USA. 103(33):12285-12290.

Buehler MJ. 2007. Hierarchical chemo-nanomechanics of stretching protein molecules: Entropic elasticity, protein unfolding and molecular fracture. J. Mech. Mater. Struct. 2(6):1019-1057.

Buehler MJ. 2007. Nano- and micromechanical properties of hierarchical biological materials and tissues. J. Mater. Sci. 42(21):8765-8770.

Buehler MJ, Ackbarow T. 2007. Fracture mechanics of protein materials. Mater. Today 10(9):46-58.

Bustamante C et al., 1994. Entropic elasticity of lambda-phage DNA. Science 265(5178):1599-1600.

Cui XQ et al., 2007. Biocatalytic generation of ppy-enzyme-CNT nanocomposite: From network assembly to film growth. J. Phys. Chem. C. 111(5):2025-2031.

Currey JD. 2002. Bones: Structure and mechanics. Princeton, NJ: Princeton University Press.

1260 Dao M, Lim CT, Suresh S. 2003. Mechanics of the human red blood cell deformed by optical tweezers. J. Mech. Phys. Solids 51(11-12):2259-2280.

Doyle J. 2007. Rules of engagement. Nature 446:860.

Dudko OK, Hummer G, Szabo A. 2006. Intrinsic rates and activation free energies from single-molecule pulling experiments. Phys. Rev. Lett. 96(10).
Duin ACTv et al., 2001. Reaxff: A reactive force field for hydrocarbons. J. Phys. Chem. A. 105:9396-9409.

Engler AJ et al., 2006. Matrix elasticity directs stem cell lineage specification. Cell 126(4):677-689.

Evans E, Ritchie K. 1997. Dynamic strength of molecular adhesion bonds. Biophys. J. 72(4):1541-1555.

Fraser P, Bickmore W. 2007. Nuclear organization of the genome and the potential for gene regulation. Nature. 447(7143): 413-417.

Fratzl P et al., 2004. Structure and mechanical quality of the collagen-mineral nano-composite in bone. J. Mater. Chem. 14(14):2115-2123.

Frost HJ, Ashby MF. 1982. Deformation-mechanism maps. Oxford: Pergamon Press.

Gao M, Lu H, Schulten K. 2002. Unfolding of titin domains studied by molecular dynamics simulations. J. Muscle Res. Cell Motil. 23(5-6):513-521.

Gilli P et al., 2004. Covalent versus electrostatic nature of the strong hydrogen bond: Discrimination among single, double, and asymmetric single-well hydrogen bonds by variabletemperature $X$-ray crystallographic methods in beta-diketone enol RAHB systems. J. Am. Chem. Soc. 126(12): 3845-3855.

Goddard WA. 2006. In: Yip S, editor. A perspective of materials

Q5 modeling in handbook of materials modeling. Springer.

Hayashi CY, Lewis RV. 1998. Evidence from flagelliform silk cDNA for the structural basis of elasticity and modular nature of spider silks. J. Mol. Biol. 275(5):773-784.

Helfand BT, Chang L, Goldman RD. 2004. Intermediate filaments are dynamic and motile elements of cellular architecture. J. Cell Sci. 117(2):133-141.

Hirth JP, Lothe J. 1982. Theory of dislocations. WileyInterscience.

Holland JH. 1995. Hidden order - how adaptation builds complexity. Reading, MA: Helix Books.

Hule RA, Pochan DJ. 2007. Polymer nanocomposites for biomedical application. MRS Bull. 32(4):5.

Kageshima $\mathrm{M}$ et al., 2001. Insight into conformational changes of a single $\alpha$-helix peptide molecule through stiffness measurements. Chem. Phys. Lett. 343(1-2):77-82.

Keten S, Buehler MJ. 2008. Geometric confinement governs the rupture strength of H-bond assemblies at a critical length scale. Nano Lett. 8(2):743-748.

Kiss B, Karsai A, Kellermayer MSZ. 2006. Nanomechanical properties of desmin intermediate filaments. J. Struct. Biol. 155(2):327-339.

Kitano H. 2002a. Computational systems biology. Nature 420(6912):206-210.

Kitano H. 2002b. Systems biology: A brief overview. Science 295(5560): $1662-1664$.

Lakes R. 1993. Materials with structural hierarchy. Nature 361(6412):511-515.

Langer R, Tirrell DA. 2004. Designing materials for biology and medicine. Nature. 428(6982):487-492.

Lantz MA et al., 1999. Stretching the $\alpha$-helix: A direct measure of the hydrogen-bond energy of a single-peptide molecule. Chem. Phys. Lett. 315(1-2):61-68.

Lim CT et al., 2006. Experimental techniques for single cell and single molecule biomechanics. Mater. Sci. Eng. C Biomimet. Supramol. Syst. 26(8):1278-1288.

Lu H, Schulten K. 1999. Steered molecular dynamics simulations of force-induced protein domain unfolding. Proteins-Struct. Funct. Genet. 35(4):453-463. 
Lu H et al., 1998. Unfolding of titin immunoglobulin domains by steered molecular dynamics simulation. Biophys. J. 75(2): $662-671$.

MacKerell AD et al., 1998. All-atom empirical potential for molecular modeling and dynamics studies of proteins. J. Phys. Chem. B. 102(18):3586-3616.

Mershin A et al., 2005. A classic assembly of nanobiomaterials. Nat. Biotechnol. 23(11):1379-1380.

Mitsui K et al., 2000. Dynamic measurement of single protein's mechanical properties. Biochem. Biophys. Res. Commun. 272(1):55-63.

Mucke $\mathrm{N}$ et al., 2004. Assessing the flexibility of intermediate filaments by atomic force microscopy. J. Mol. Biol. 335(5): $1241-1250$.

Nelson MT et al., 1996. NAMD: A parallel, object oriented molecular dynamics program. Int. J. Supercomput. Appl. High Perform. Comput. 10(4):251-268.

Nieh TG, Wadsworth J. 1991. Hall-Petch relation in nanocrystalline solids. Scripta Met. 25(4).

Petka WA et al., 1998. Reversible hydrogels from selfassembling artificial proteins. Science 281(5375):389-392.

Prater CB, Butt HJ, Hansma PK. 1990. Atomic force microscopy. Nature 345(6278):839-840.

Ramachandran GN, Kartha G. 1955. Structure of collagen. Nature 176:593-595.

Sarikaya M et al., 2003. Molecular biomimetics: Nanotechnology through biology. Nat. Mater. 2(9):577-585.

Schwaiger I et al., 2002. The myosin coiled-coil is a truly elastic protein structure. Nat. Mater. 1(4):232-235.

Sheu S-Y et al., 2003. Energetics of hydrogen bonds in peptides. Proc. Natl. Acad. USA. 100(22):12683-12687.

Smeenk JM et al., 1971. Controlled assembly of macromolecular beta-sheet fibrils. Angew. Chem. Int. Ed. 44(13):1968-1971.

Smith BL et al., 1999. Molecular mechanistic origin of the toughness of natural adhesives, fibres and composites. NatureQ7 399(6738):761-763.
Sotomayor M, Schulten K. 2007. Single-molecule experiments in vitro and in silico. Science 316(5828):1144-1148.

Sun YL et al., 2004. Stretching type II collagen with optical tweezers. J. Biomech. 37(11):1665-1669.

Tai K, Ulm FJ, Ortiz C. 2006. Nanogranular origins of the strength of bone. Nano Letters 11:2520-2525.

Taylor GI. 1934. Mechanism of plastic deformation in crystals. Proc. R. Soc. A. 145:362.

Wang N, Stamenovic D. 2002. Mechanics of vimentin intermediate filaments. J. Muscle Res. Cell Motil. 23(5-6): $535-540$.

Wang W et al., 2001. Biomolecular simulations: Recent developments in force fields, simulations of enzyme catalysis, protein-ligand, protein-protein, and proteinnucleic acid noncovalent interactions. Annu. Rev. Biophys. Biomol. Struct. 30:211-243.

Wegst UGK, Ashby MF. 2004. The mechanical efficiency of natural materials. Philos. Mag. 84(21):2167-2181.

Weiner S, Wagner HD. 1998. The material bone: Structure mechanical function relations. Annu. Rev. Mater. Sci. 28:271-298.

Wiita AP et al., 2006. Force-dependent chemical kinetics of disulfide bond reduction observed with single-molecule techniques. Proc. Natl. Acad. Sci. USA. 103(19):7222-7227.

Winey KI, Vaia RA. 2007. Polymer nanocomposites. MRS Bull. 32(4):5.

Wolf D et al., 2003. Deformation mechanism and inverse HallPetch behavior in nanocrystalline materials. Z. Metallk 94: 1052-1061.

Yip S. 1998. The strongest size. Nature 391:532-533.

Zhao XJ, Zhang SG. 2006. Molecular designer self-assembling peptides. Chem. Soc. Rev. 35(11):1105-1110.

Zhao XJ, Zhang SG. 2007. Designer self-assembling peptide materials. Macromol. Biosci. 7(1):13-22. 